\title{
Differentiation of genetically modified canine bone mesenchymal stem cells labeled with superparamagnetic iron oxide into neural-like cells
}

\author{
XING-LONG LIU*, QING-QUAN ZU* , BIN WANG, SHAN-SHAN LU, \\ XIAO-QUAN XU, SHENG LIU and HAI-BIN SHI \\ Radiology Department, The First Affiliated Hospital of Nanjing Medical University, \\ Jiangsu Province Hospital, Nanjing, Jiangsu 210029, P.R. China
}

Received August 28, 2017; Accepted December 19, 2017

DOI: $10.3892 / \mathrm{mmr} .2018 .8847$

\begin{abstract}
The use of mesenchymal stem cells (MSCs) has been reported to improve outcomes in various types of nervous system diseases, primarily based on their neural regenerative differentiation ability and paracrine effect on different neuroprotective cytokines. Genetically modified MSCs may enhance the paracrine effect and may further improve the cell-based therapeutic outcome of nervous system diseases. Magnetic resonance imaging has been used to monitor distribution and migration of cells labeled with superparamagnetic iron oxide (SPIO) nanoparticles. However, few studies have described the neural differentiation ability of genetically modified and SPIO-labeled MSCs, which is the foundation for cell tracking and cell therapy in vivo. In this study, canine bone marrow-derived MSCs (BMSCs) were initially labeled with SPIO, by culturing with $20 \mu \mathrm{g} / \mathrm{ml}$ SPIO for $24 \mathrm{~h}$, and transfected with the brain-derived neurotrophic factor (BDNF) gene using lentivirus transfection at different multiplicities of infection (MOI) values. The optimized MOI value was demonstrated by cellular viability and enhanced green fluorescent protein (eGFP) rate. Subsequently, the BMSCs were induced to differentiate into neuron-like cells by chemical induction. The results demonstrated that BDNF-overexpressing BMSCs labeled with SPIO can be induced into neuron-like cells with high efficiency and minimal effects on cell viability. Additionally, following
\end{abstract}

Correspondence to: Dr Sheng Liu or Dr Hai-Bin Shi, Radiology Department, The First Affiliated Hospital of Nanjing Medical University, Jiangsu Province Hospital, 300 Guangzhou Road, Nanjing, Jiangsu 210029, P.R. China

E-mail: shihb@njmu.edu.cn

E-mail: liusheng@njmu.edu.cn

*Contributed equally

Key words: bone marrow-derived mesenchymal stem cells, brain-derived neurotrophic factor, superparamagnetic iron oxide, neural differentiation neural differentiation, the cells transfected with BDNF and labeled with SPIO expressed significantly higher levels of BDNF and neural markers. The overexpression of BDNF may contribute to neural differentiation of BDNFs, and may have potential benefits for further BMSC-based therapy in vivo.

\section{Introduction}

Mesenchymal stem cells (MSCs) have been used as a powerful stem cell source for cellular transplantation therapy, as they exhibit numerous advantages (1-4). MSCs may be easily obtained from various types of tissues, and differentiate into different cell and tissue types with immunomodulatory properties (5) and homing capacity (6). MSCs produce cytokines and chemokines that may promote cell proliferative, anti-apoptotic, anti-inflammatory and cell homing effects in wounded areas $(7,8)$. Brain-derived neurotrophic factor (BDNF) is a cytokine secreted by MSCs and has neuroprotective, neurogenic and angiogenic effects, thus promoting recovery after central nervous system (CNS) insult $(9,10)$. Modified MSCs overexpressing BDNF may theoretically improve their therapeutic effect.

Although certain studies using genetically modified techniques have exhibited promising results in cell culture and small-animal CNS insult models, further preclinical research is required in large animals due to numerous discordances between laboratory and clinical research (11). Additionally, the labeling of superparamagnetic iron oxide (SPIO) nanoparticles of cells is a way of tracing the distribution and migration of transplanted cells in large animals by magnetic resonance imaging (MRI) in vivo with minor effects on cell viability $(12,13)$. However, few studies have reported the efficiency of genetically modified MSCs after labeling with SPIO, especially for large animals such as canines, which may be used as a suitable model of human neurological disease and is evaluated by MRI $(12,14)$.

Therefore, in this study, SPIO-labeled canine BMSCs were used to evaluate the feasibility of BDNF gene lentiviral transfection and the neural differentiation efficiency after gene modification. 


\section{Materials and methods}

Cell culture and identification. Bone marrow was extracted from the humerus of 20 healthy adult beagle dogs of either sex (sex ratio, males to females $3: 1$ ) and $14.62 \pm 1.1 \mathrm{~kg}$ body weight (Laboratory Animal Centre, Anhui, China) as previously described (12). All dogs were housed in a single cages separately at well-ventilated facility with purified air and $12 \mathrm{~h}$ light/dark cycle at $24-28^{\circ} \mathrm{C}$ and fed twice a day with commercial dry food and sterile water ad libitum. The National Institutes of Health (Bethesda, MD, USA) and Institutional Animal Care and Use Committee (IACUC) guidelines for use of animals in research were followed, under an approved IACUC protocol of Nanjing Medical University (Nanjing, China). MSCs were isolated and purified from the bone marrow by density gradient centrifugation and plastic wall adherence. Briefly, the bone marrow was layered onto a density gradient solution at 1:1 volume ratio with equal volume (Ficoll-Paque; Tianjin Haoyang Biological Products Technology Co., Ltd., Tianjin, China), and centrifuged for $20 \mathrm{~min}$ at $400 \mathrm{x}$ g at $4^{\circ} \mathrm{C}$. Enriched cells were obtained from the solution interphase and washed twice with PBS, then cultivated in normal culture medium: Low-glucose Dulbecco's modified Eagle medium (DMEM; Gibco; Thermo Fisher Scientific, Inc., Waltham, MA, USA) supplemented with $10 \%$ fetal bovine serum (FBS; Gibco; Thermo Fisher Scientific, Inc.), $100 \mathrm{IU} / \mathrm{ml}$ penicillin and $100 \mu \mathrm{g} / \mathrm{ml}$ streptomycin at $37^{\circ} \mathrm{C}$ and $5 \% \mathrm{CO}_{2}$. Primary isolated MSCs were defined as passage (P)0. When $80-90 \%$ confluence was reached, the cells were passaged (1:2 or 1:3 dilutions) with fresh medium.

To determine the expression of MSC markers [cluster of differentiation (CD)34-, CD45-, CD44+ and CD90+], P2 BSMCs were used for flow cytometry (BD FACSCalibur; BD Biosciences, Franklin Lakes, NJ, USA). In brief, BMSCs were collected in FACS tubes (BD Biosciences) at $1 \times 10^{6}$ cells/tube and washed with PBS. Cells were incubated with antibodies at room temperature for $1 \mathrm{~h}$. The antibodies included: anti-CD34 (cat. no. ab81289), CD45 (cat. no. ab123522), CD44 (cat. no. ab157107), CD90 (cat. no. ab123511; all Abcam, Cambridge, UK) all at 1:200. Labeled cells were washed twice with PBS and re-suspender in $500 \mu \mathrm{l}$ FACS buffer. Subsequently, the cells were incubated with rabbit anti-mouse immunoglobulin $\mathrm{G}$ secondary antibody labeled with phycoerythrin $(1: 1,000$; cat. no. ab7000; Abcam) of for $1 \mathrm{~h}$. The expression of these markers was evaluated using FlowJo software (version 7.6.3; FlowJo LLC, Ashland, OR, USA) for data analysis.

To determine the multiple differentiation capacity, P3 BMSCs were induced to osteoblastic and adipose differentiation as previously reported (1). For osteogenic differentiation, cells were seeded at a density of $4.5 \times 10^{4}$ cells $/ \mathrm{cm}^{2}$ and cultured with induction medium containing DMEM supplemented with 10\% FBS (both Gibco; Thermo Fisher Scientific, Inc.), $100 \mathrm{nM}$ dexamethasone, $10 \mathrm{mM} \beta$-glycerol-phosphate, and $100 \mu \mathrm{g} / \mathrm{ml}$ ascorbic acid (all purchased from Sigma-Aldrich; Merck KGaA, Darmstadt, Germany) at $37^{\circ} \mathrm{C}$ and $5 \% \mathrm{CO}_{2}$. The induction medium was changed every 3 days. After 14 days of induction, cells were fixed in $70 \%$ ethanol and stained with $2 \%$ Alizarin Red at room temperature for $10 \mathrm{~min}$. For adipogenic differentiation, cells were plated at a density of $1.0 \times 10^{4}$ cells $/ \mathrm{cm}^{2}$. Similarly, adipogenesis was induced by culturing with induction medium containing DMEM supplemented with 5\% rabbit serum (Gibco; Thermo Fisher Scientific, Inc.), $5 \mu \mathrm{g} / \mathrm{ml}$ insulin, $1 \mu \mathrm{M}$ dexamethasone and $5 \mu \mathrm{g} / \mathrm{ml}$ rosiglitazone (all obtained from Sigma-Aldrich; Merck $\mathrm{KGaA}$ ) at $37^{\circ} \mathrm{C}$ and $5 \% \mathrm{CO}_{2}$. The induction medium was changed every 3 days. After 21 days of induction, cells were fixed in $70 \%$ ethanol and stained with $0.3 \%$ Oil Red $\mathrm{O}$ at room temperature for $60 \mathrm{~min}$.

SPIO labeling. BMSCs were cultured in $10 \mathrm{~cm}$ dish at $2 \times 10^{6}$ with fresh medium containing SPIO- poly-L-lysine (Nanjing Nanoeast Biotech, Co., Ltd., Nanjing, China) at $20 \mu \mathrm{g} / \mathrm{ml}$ for $24 \mathrm{~h}$ and then were washed three times in PBS to eliminate extracellular SPIO. Efficiency of SPIO labeling was confirmed by Prussian blue (PB) staining (12). In brief, the cells were incubated with PB (2\% potassium ferrocyanide in $6 \%$ hydrogen chloride) for $15 \mathrm{~min}$ at room temperature and then counterstained with nuclear fast red for $1 \mathrm{~min}$ at room temperature. The percentage of iron-containing cells was calculated according to the positively-stained cell numbers with light microscope (IX71; Olympus Corp., Tokyo, Japan).

Lentivirus transfection. SPIO-BMSCs were seeded in 96-well plates at $1 \times 10^{4}$ cells per well $(100 \mu \mathrm{l})$, cultured overnight and transfected with enhanced green fluorescent protein (eGFP) (+)-BDNF(-)-lentivirus (Shanghai GeneChem, Co., Ltd., Shanghai, China) at a multiplicity of infection (MOI) of $0,1,5$, $10,20,50$ and 100 . After $12 \mathrm{~h}$, transfection medium was changed to normal culture medium without lentiviral particles, and cells were cultured as routine. MSCs were used for analysis at day 3, 7 and 14. The percentage eGFP-positive cells was detected by Guava Express analysis on a GuavaEasyCyte ${ }^{\mathrm{TM}}$ flow cytometer (Guava Technologies; EMD Millipore, Billerica, MA, USA). At each time point, the cellular viability was evaluated by using a Cell Counting kit-8 (CCK-8; Dojindo Molecular Technologies, Inc., Kumamoto, Japan). In brief, $10 \mu \mathrm{l}$ CCK-8 was added into each well and after $4 \mathrm{~h}$ incubation, and the optical density value was measured at a wavelength of $450 \mathrm{~nm}$ using a microplate reader (Thermo Fisher Scientific, Inc.). The optimal MOI was calculated by multiplication of the percentage eGFP positive cells by the average cellular survival rate.

BMSC neural differentiation. After determination of the optimal MOI, SPIO labeled MSCs were divided into 4 groups: Transfection at optimized MOI with eGFP(-)-BDNF(+)-lentivirus (BDNF+ group), transfection at optimized MOI with eGFP(-)-BDNF(-)-lentivirus (BDNFgroup) and non-transfected group (mock group), for neural differentiation and subsequent analysis. The non-transfected group without neural differentiation was used as control group (control group). Neural differentiation was induced as previously reported $(15,16)$ in $\mathrm{BDNF}+, \mathrm{BDNF}$ - and mock group cells. Briefly, BMSCs were cultured with DMEM $+10 \%$ FBS until sub-confluence. BMSCs were pre-induced for $24 \mathrm{~h}$ with $\mathrm{DMEM}+20 \% \mathrm{FBS}+1 \mathrm{mM} \beta$-mercaptoethanol, then were induced for $8 \mathrm{~h}$ with DMEM $+100 \mathrm{mM}$ butylated hydroxyanisole $(\mathrm{BHA})+2 \%$ dimethylsulfoxide (DMSO). Finally, of the media of the cells was replaced with maintenance media containing DMEM + $100 \mathrm{mM} \mathrm{BHA}+2 \% \mathrm{DMSO}+25 \mathrm{mM}$ $\mathrm{KCl}+2 \mathrm{mM}$ valproic acid $+10 \mu \mathrm{M}$ forskolin $+\mathrm{N}_{2}$ supplement 
Table I. Primers of canine specific markers.

\begin{tabular}{|c|c|c|}
\hline Gene & Primer sequence & Marker \\
\hline Nestin & $\begin{array}{l}\text { F: TGTAGGAGGTCCCTAAGCCC } \\
\text { R: CACATCCCTACACCACACCC }\end{array}$ & Neural progenitor cell \\
\hline TUJ1 & $\begin{array}{l}\text { F: GCACACTGCTCATCAACAAG } \\
\text { R: TCTTGCTCTCCTTCATGGAC }\end{array}$ & Neuron \\
\hline GFAP & $\begin{array}{l}\text { F: TGAGATCGCCACCTACAGGA } \\
\text { R: ATCCAACACCTTGCCCACAA }\end{array}$ & Astrocyte \\
\hline BDNF & $\begin{array}{l}\text { F: CGCGGACTTGTATACCTCCC } \\
\text { R: GGGACTTTTTCGAGGACGGT }\end{array}$ & Neurotrophin \\
\hline VEGF & $\begin{array}{l}\text { F: CCCGGTATAAACCCTGGAGC } \\
\text { R: ACGCGAGTCTGTGTTTTTGC }\end{array}$ & Angiogenesis \\
\hline CXCR-4 & $\begin{array}{l}\text { F: CAGTTGAGGCTGTGGCAAAC } \\
\text { R: GAGAGCAGGTATCCAGACGC }\end{array}$ & Chemokine \\
\hline GUSB & $\begin{array}{l}\text { F: ACATCGACGACATCACCGTCA } \\
\text { R: GGAAGTGTTCACTGCCCTGGA }\end{array}$ & Housekeeping gene \\
\hline
\end{tabular}

Housekeeping gene GUSB was used as internal control gene. TUJ1, class $3 \beta$ tubulin; GFAP, glial fibrillary acidic protein; BDNF, brain-derived neurotrophic factor; VEGF, vascular endothelial growth factor; CXCR-4, chemokine receptor type 4; GUSB, glucuronidase $\beta$.

for 1-3 days. BMSCs in the control group were cultured with $\mathrm{DMEM}+10 \%$ FBS.

Reverse transcription-quantitative polymerase chain reaction (RT-qPCR). Expression of cytokines [(BDNF, vascular endothelial growth factor (VEGF) and chemokine receptor type 4 (CXCR-4)] and neural precursor cells (indicated by nestin expression), neurons [indicated by class $3 \beta$ tubulin (TUJ1) expression], neurogliocytes [indicated by glial fibrillary acidic protein (GFAP) expression] and oligodendrocytes (oligo1) were analyzed by RT-qPCR. Total RNA was extracted from cultured cells using TRIzol reagent (Thermo Fisher Scientific, Inc.) according to the manufacturer's protocol. RNA was used for cDNA synthesis using a Prime Script RT Reagent kit according to the manufacture's protocol and oligo dT primers (Takara Biotechnology, Co., Ltd., Dalian, China). Briefly, the first-strand cDNA was obtained by reverse transcription using $3 \mu \mathrm{l}$ of total RNA at $37^{\circ} \mathrm{C}$ for $15 \mathrm{~min}$ and $85^{\circ} \mathrm{C}$ for $5 \mathrm{sec}$. RT-qPCR was performed at $95^{\circ} \mathrm{C}$ for $5 \mathrm{sec}$ and $60^{\circ} \mathrm{C}$ for $34 \mathrm{sec}$ in $20 \mu \mathrm{l}$ buffer containing SYBR premix ExTaq II and ROX Reference dye (Takara Biotechnology, Co., Ltd.) and $0.2 \mu \mathrm{M}$ each of the primers and SYBR Premix DimerEraser (Takara Biotechnology, Co., Ltd.) on a 7900HT system (ABI Prism ${ }^{\circledR}$ 7900HT; Thermo Fisher Scientific, Inc). Glucuronidase $\beta$ was used as an internal control. The relative level of gene expression for each sample was calculated using the $2^{-\Delta \Delta \mathrm{Cq}}$ method (17). Primer sequences are listed in Table I.

Immunofluorescence. To confirm the differentiation of the BMSCs, neuron-like cells were analyzed by immunofluorescence using antibodies against nestin, GFAP and TUJ1. The primary antibodies included mouse anti-nestin (1:200; cat. no. ab22035), mouse anti-GFAP (1:200; cat. no. ab53554), rabbit anti-nestin (1:200; cat. no. ab105389) and rabbit anti-class $3 \beta$ tubulin (1:500; cat. no. ab7751; all Abcam). Cells were fixed with $4 \%$ paraformaldehyde at room temperature for $20 \mathrm{~min}$. Cells were washed three times with PBS and blocked with $0.4 \%$ Triton X-100 (Sigma-Aldrich; Merck KGaA) at $4^{\circ} \mathrm{C}$ for $10 \mathrm{~min}$. Then cells were washed three times with PBS and immersed with $5 \%$ bovine serum albumin at $37^{\circ} \mathrm{C}$ for $30 \mathrm{~min}$. Following incubation with the primary antibodies overnight at $4^{\circ} \mathrm{C}$, the cells were washed three times with PBS, followed by incubation with fluorescent goat anti-mouse immunoglobulin $\mathrm{G}$ (IgG) antibody (cat no. ab150113), goat anti-mouse IgG antibody (cat no. ab97035), goat anti-rabbit IgG (cat no. ab150079) and goat anti-rabbit IgG (cat no. ab6787; all Abcam) at room temperature for $60 \mathrm{~min}$, all at 1:200. Following washing, the cells were stained with $100 \mathrm{ng} / \mathrm{ml}$ DAPI for $10 \mathrm{~min}$ at room temperature and mounted with anti-fade mounting medium (both Sigma-Aldrich; Merck KGaA), and observed under a fluorescent microscope. BMSCs that expressed neural (neuron or glia) markers were considered to be immunopositive cells.

Statistical analysis. All analyses were performed in a double-blinded manner. All values are expressed as the mean \pm standard deviation. Student's t-test and one-way analysis of variance followed by Bonferroni's post hoc analysis were used for single and multiple comparisons, respectively. Data was calculated using SPSS software version 17.0 (SPSS, Inc., Chicago, IL, USA). $\mathrm{P}<0.05$ was considered to indicate a statistically significant difference.

\section{Results}

Morphology and phenotypes of cultured BMSCs. Following isolation by density gradient centrifugation from canine bone marrow, BMSCs were cultured in growth medium. After 14 days of culturing, the cell morphology was altered 

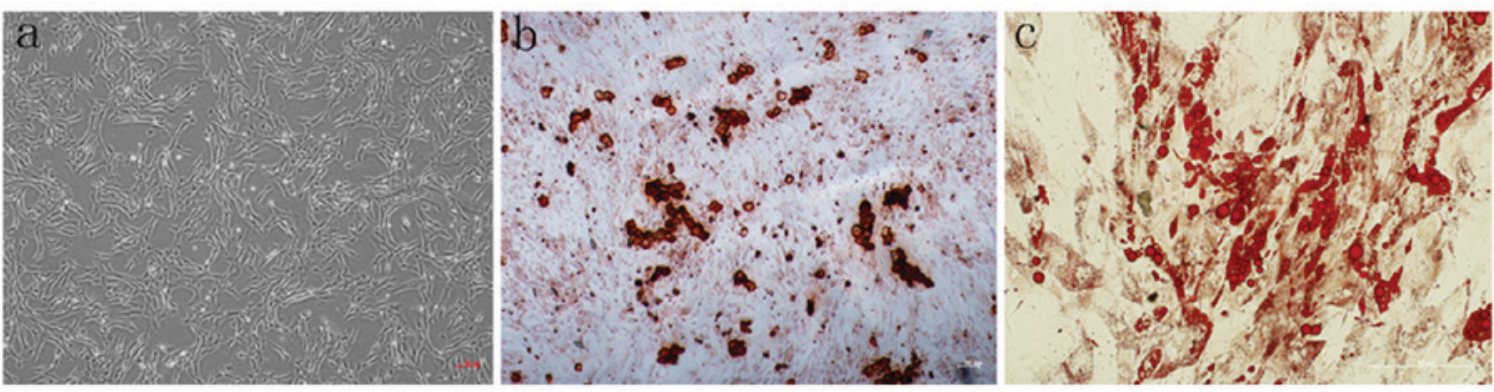

Figure 1. Osteogenic and adipogenic differentiation of BMSCs. (A) Passage 3 BMSCs prior to differentiation (magnification, x40). (B) Red osteal nodules were observed by slizarin red staining after 14 days of osteogenic differentiation (magnification, x100). (C) Red lipid droplets were observed by Oil red O staining after 21 days of adipogenic differentiation (magnification, x200). BMSCs, bone marrow-derived mesenchymal stem cells.
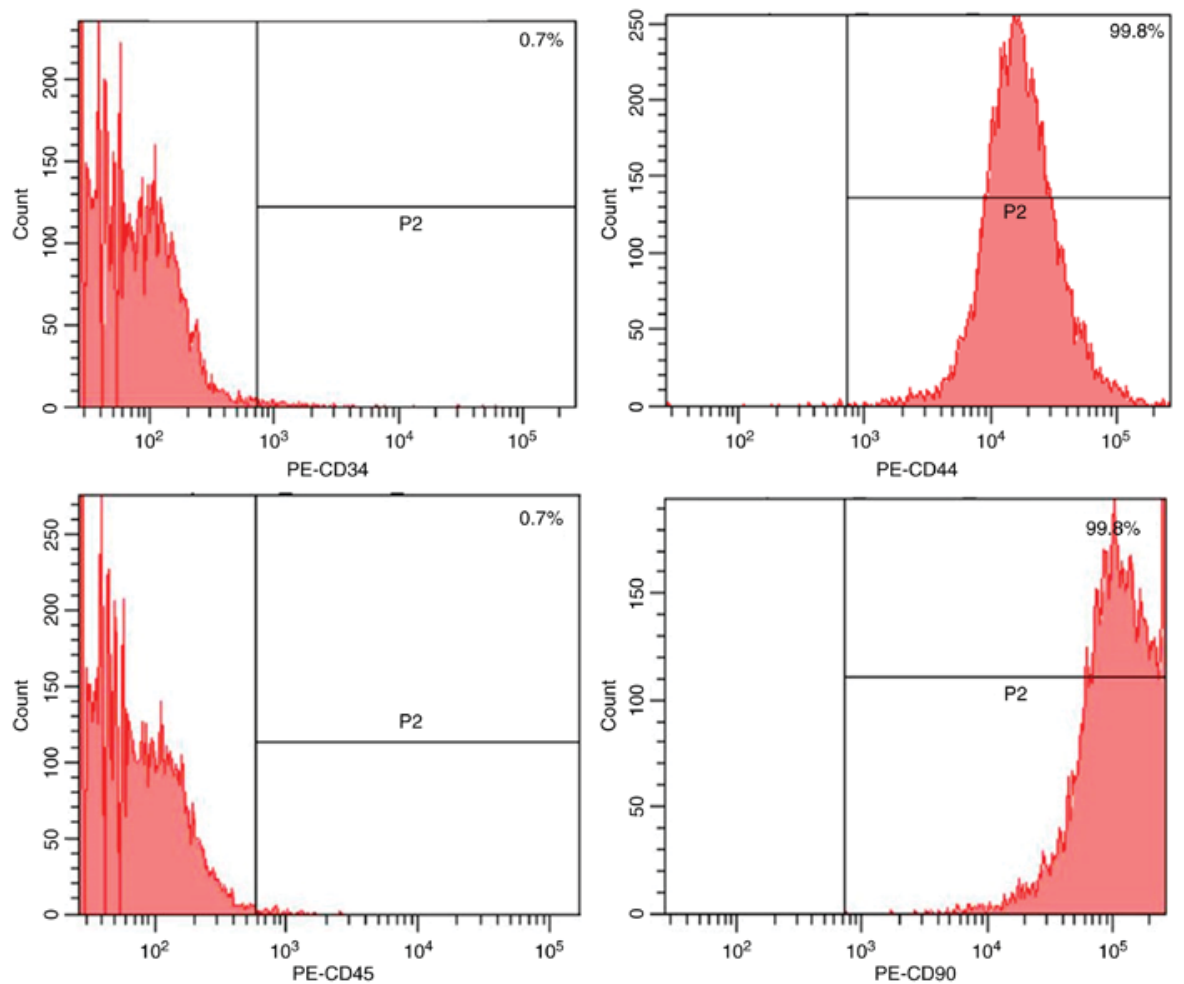

Figure 2. Flow cytometry analysis of BMSC surface markers. Flow cytometry analysis presents the MSC-positive surface markers CD44 and CD90 expressed in $99.8 \%$ of cells, whereas the MSC-negative surface markers CD34 and CD45 are expressed in $0.7 \%$ of cells. BMSCs, bone marrow-derived mesenchymal stem cells; $\mathrm{CD}$, cluster of differentiation.

from various shapes including discoidal flat, triangular and elongated, to a spindle shape under a light microscope (Fig. 1a). After 14 days of osteogenic differentiation, the cells differentiated into the osteogenic lineage, as demonstrated by slizarin red staining (Fig. 1b). Additionally, after 21 days of adipogenic differentiation, these cells differentiate into adipocytes with lipid droplets stained with Oil red O (Fig. 1c). Flow cytometry analysis (Fig. 2) revealed that passage 2 BMSCs (7-day culture) were positive for CD44 and CD90 and negative for CD34 and CD45.

SPIO labeling. The uptake mechanism of SPIO particles by BMSCs is mediated by endocytosis. In a previous study, $20 \mu \mathrm{g} / \mathrm{ml}$ was chosen as the optimal concentration for high uptake efficiency and minimal cytotoxicity (12). After incubation with SPIO for 24 h, SPIO particles (blue staining; Fig. 3a) were visible following PB staining, and the labeling percentage of SPIO was $\sim 100 \%$ (Fig. 3a).

Optimized MOI of lentivirus transfection. After incubation with eGFP(+)-BDNF(-)-lentivirus for $12 \mathrm{~h}$, SPIO-labeled BMSCs (Fig. 3a) exhibited positive eGFP expression on day 3 (Fig. 3b), and proliferated with positive eGFP expression on day 7 (Fig. 3c). As demonstrated in Fig. 4, the eGFP expression rate was MOI dose-dependent at day 3 and exhibited a $\geq 80 \%$ positive rate when MOI $\geq 5$ was used at day 14 . However, with the increasing of MOI, the cellular survival rate decreased (MOI 50 and 100). Following combining the eGFP positive rate and the average cellular survival rate at different MOIs, it was revealed that at MOI 10, BMSCs exhibited higher eGFP expression and proliferation capacity at day 7 and this was maintained at day 14 , (\% eGFP x\% survival cells $\times 10^{4}$ 

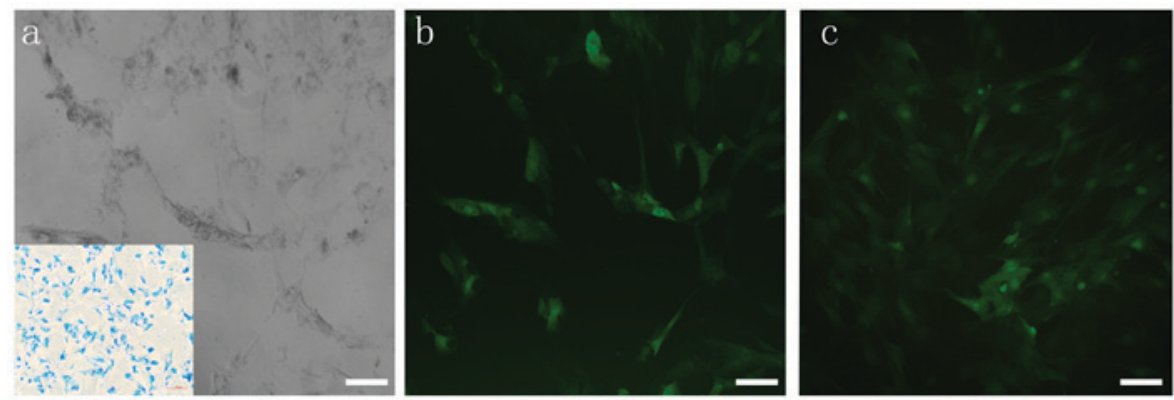

Figure 3. Photomicrography of BMSCs following incubation with SPIO-PLL and transfection with eGFP + lentivirus. (a) SPIO particles were observed under a light microscope. The left bottom panel presents Prussian blue staining, which represents the SPIO particles inside BMSCs. (b) Fluorescence microscopy revealed the expression of eGFP at day 3. (c) Proliferation of SPIO labeled and lentivirus transfected BMSCs at day 7. Scale bar, $50 \mu \mathrm{m}$. eGFP, enhanced green fluorescent protein; BMSCs, bone marrow-derived mesenchymal stem cells; SPIO-PLL, superparamagnetic iron oxide-poly-L-lysine.
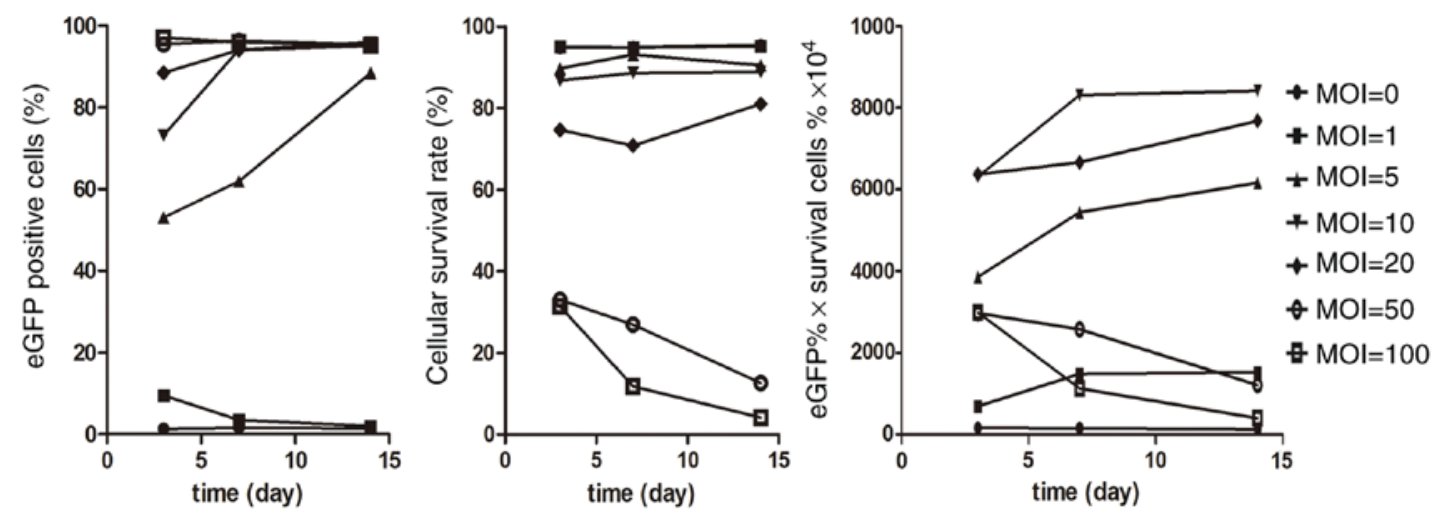

day 7
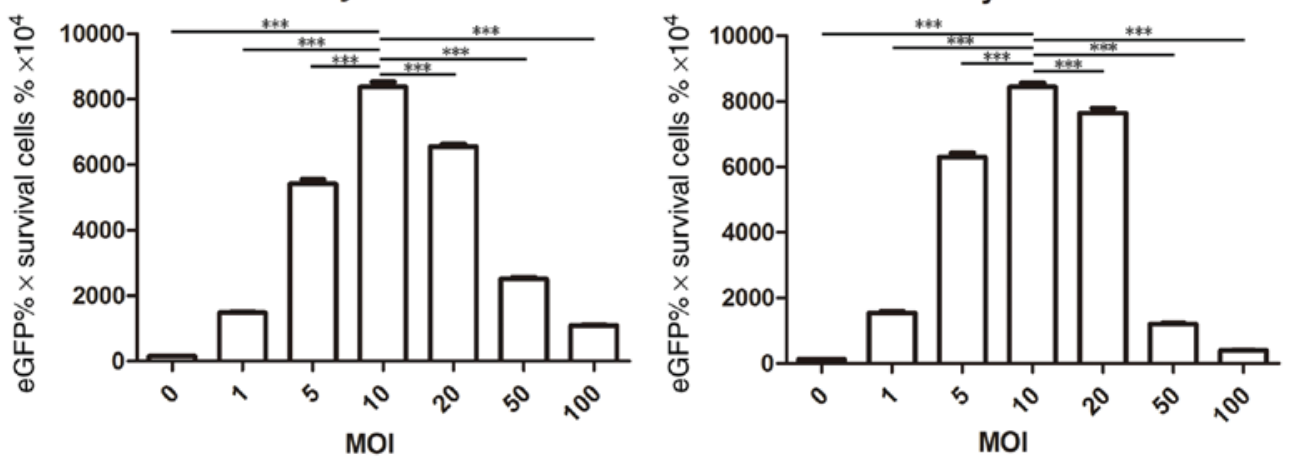

Figure 4. Flow cytometry and Cell Counting kit- 8 analysis of BMSCs following incubation with SPIO-PLL and transfection with eGFP + lentivirus. The upper left panel presents the dose-dependent effect on eGFP positive cells at day 3, and at MOI 100, the majority of BMSCs were eGFP positive, whereas at MOI 0 , eGFP was rarely expressed. The eGFP expression rate increased to $\geq 80 \%$ from day 7 to 14 at $\mathrm{MOI} \geq 5$. The upper middle graph presents the cellular survival rate. MOI $\leq 10$ maintained $\geq 80 \%$ survival rate at day $3-14$, whereas at $\mathrm{MOI} \geq 50$, the survival rate decreased from day $3-14$. The upper right graph shows the product of the eGFP positive rate and the average cellular survival rate. The lower graphs represent the significance of day 7 and day 14 , respectively, which indicated the optimal MOI was MOI 10. BMSCs, bone marrow-derived mesenchymal stem cells; SPIO-PLL, superparamagnetic iron oxide-poly-L-lysine; eGFP, enhanced green fluorescent protein; MOI, multiplicity of infection; ${ }^{* * *} \mathrm{P}<0.0001$

were $8752.37 \pm 242.11$ and $8464.76 \pm 273.40$ at day 7 and 14 respectively; $\mathrm{P}<0.0001)$.

Expression of BDNF and neural markers. To determine the expression of cytokines and neural markers following SPIO-BMSCs neural differentiation, the expression levels of cytokines (BDNF, VEGF and CXCR-4), and neural precursor cells (nestin), neurons (TUJ1) and neurogliocytes (GFAP) were detected. As exhibited in Fig. 5, following neural differentiation, the neural markers (nestin, TUJ1 and GFAP) were significantly increased in the differentiation groups compared with the control group. Additionally, VEGF and CXCR-4 were also increased markedly in the differentiation groups compared with the control, which are important for vascular formation and cell homing, respectively. The expression of BDNF was not increased following neural differentiation in the BDNF- and the mock groups; however, in the BDNF+ group, the expression of BDNF was significantly increased compared with the mock group, which suggested that there was positive gene expression during neural differentiation. Although not significant, the expression of VEGF and CXCR-4 in the BDNF+ group was 

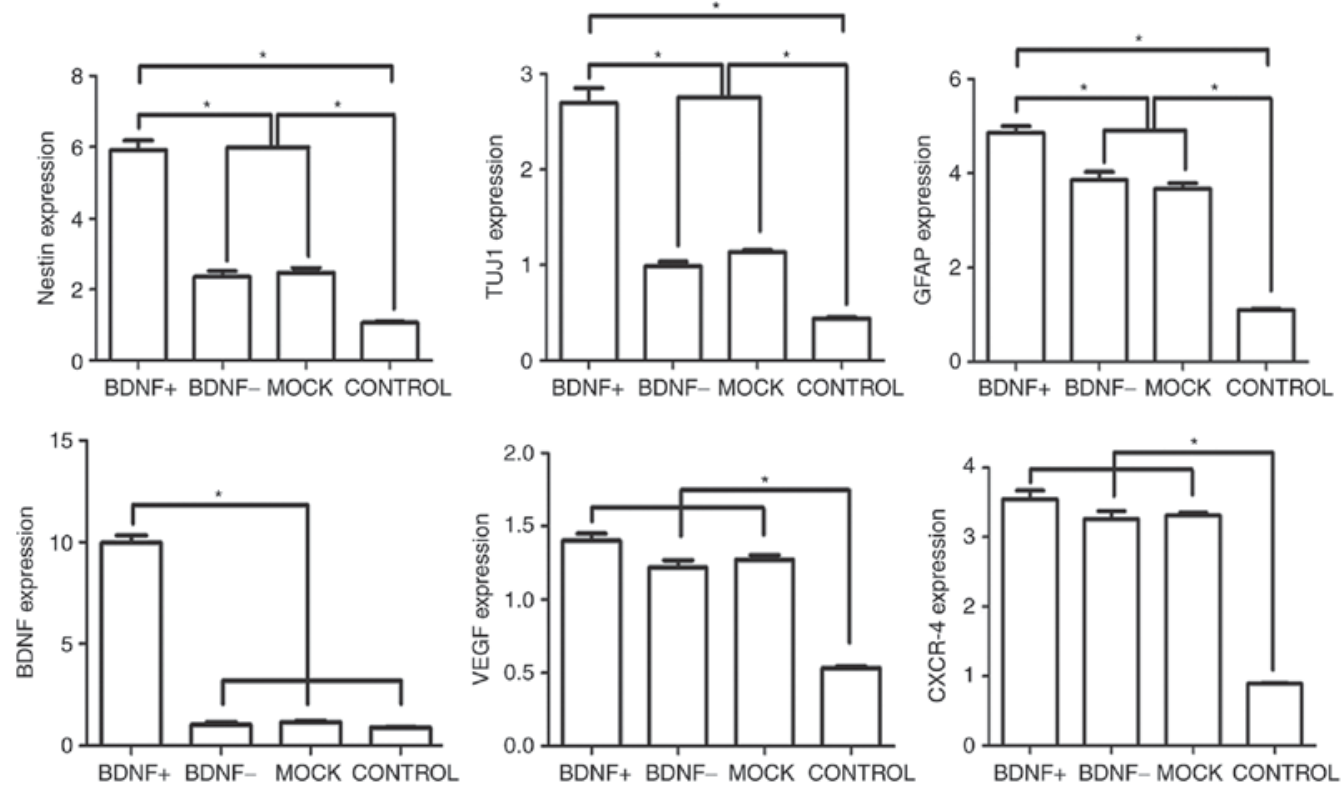

Figure 5. Reverse transcription-quantitative polymerase chain reaction analysis of BMSCs following neural differentiation. Following neural differentiation, the BDNF+ group exhibited significantly higher expression of neural markers, nestin, TUJ1 and GFAP compared with the other groups, and the expression of BDNF was also markedly higher in the BDNF+ group than the other three groups. Compared with the control group, the neural markers, VEGF and CXCR-4 expression were significantly higher in the neural differentiation groups compared with the control group. BMSCs, bone marrow-derived mesenchymal stem cells; BDNF, brain-derived neurotrophic factor; TUJ1, class $3 \beta$ tubulin; GFAP, glial fibrillary acidic protein; VEGF, vascular endothelial growth factor; CXCR-4, chemokine receptor type $4 .{ }^{*} \mathrm{P}<0.05$.

also higher than the other differentiated groups. The relative percentages of expression of neural markers and cytokines in the BDNF+, BDNF- and mock groups were evaluated (Fig. 6). The percentage of expression was calculated as follows: Expression in differentiation groups - the expression in the control group)/the expression in control group $x 100$. When comparing the BDNF+ group with the other two groups, the percentage BDNF expression was $\sim 10$ fold higher. Expression of neural markers of nestin, TUJ1 and GFAP also increased markedly in the BDNF+ group compared with BDNF- and mock groups (approximately 3-6 fold). Furthermore, expression of VEGF and CXCR-4 also increased in the BDNF+ group but the difference was not significant compared with BDNF- and mock groups.

Immunofluorescence of neural-like cells. As is presented in Fig. 7, following neural differentiation, the morphology of BMSCs changed to neuron-like cells with comprehensive axon-like connection with surrounding cells (Fig. 7). Additionally, the BDNF+ group presented with much more neuron-like cells than the other groups. Immunofluorescence revealed positive co-staining of nestin + TUJ1, nestin + GFAP and TUJ1 + GFAP (Fig. 8), and double-positive cells in the BDNF+ group was higher than the other groups, which is in accordance with the result of RT-qPCR analysis.

\section{Discussion}

The present study revealed that canine-derived SPIO-labeled and BDNF gene-modified BMSCs differentiated into neuron-like cells in vitro. In addition, following neural differentiation, the BDNF+ BMSCs had significantly

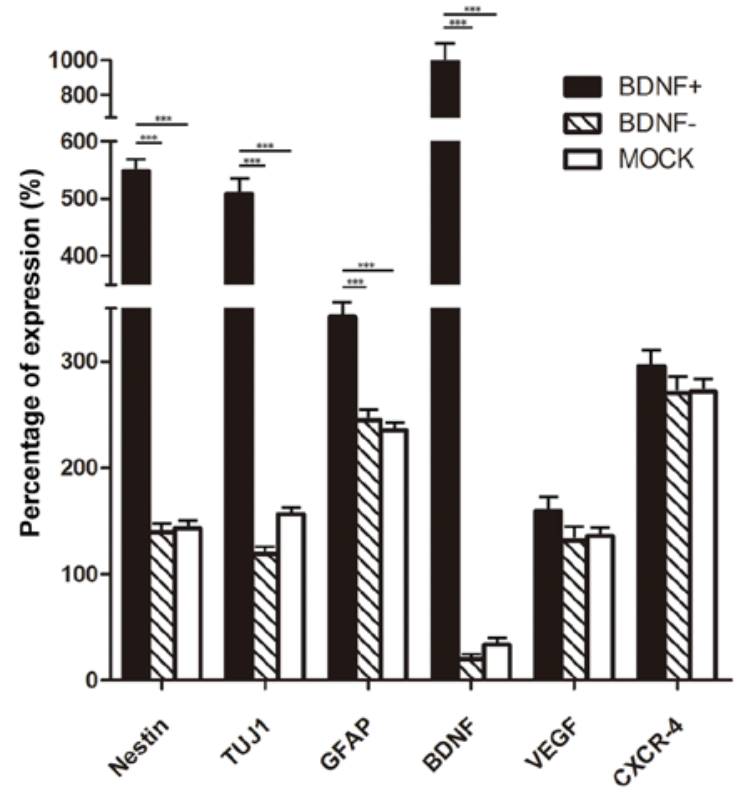

Figure 6. Percentage increases of expression neural markers and cytokines in neural differentiation groups. The neural markers and cytokines were expressed at similar levels in the BDNF- group and mock group; however, the percentage of cells expressing neural markers and BDNF was $\geq 300 \%$ (especially for BDNF, which was $\sim 1,000 \%$ ) in the BDNF+ group, which were significantly higher than BDNF- group and mock group $(\mathrm{P}<0.0001)$. BDNF, brain-derived neurotrophic factor; TUJ1, class $3 \beta$ tubulin; GFAP, glial fibrillary acidic protein; VEGF, vascular endothelial growth factor; CXCR-4, chemokine receptor type $4 .{ }^{* * *} \mathrm{P}<0.0001$.

increased expression levels of nestin, TUJ1 and GFAP, which represent neural precursor cells, neuron and neurogliocytes, respectively. These results suggested that overexpression of BDNF promotes neural differentiation, which may provide 

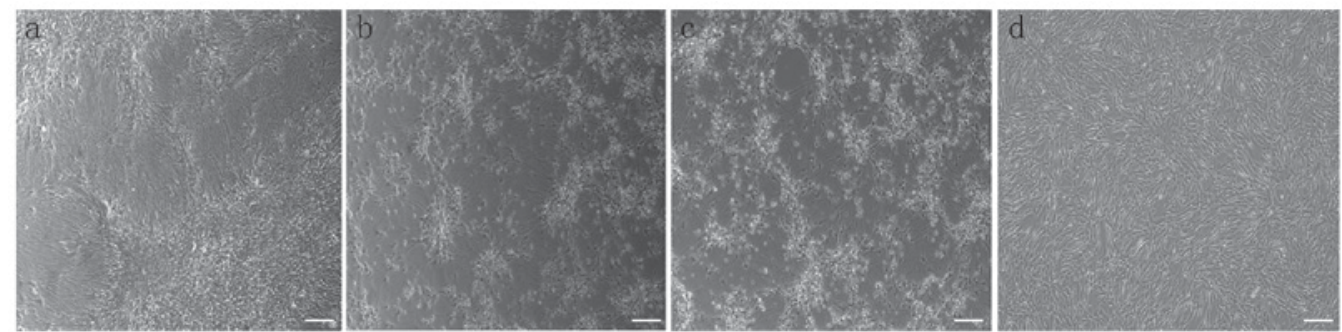

Figure 7. Photomicrography of BMSCs following neural differentiation. Following neural differentiation, the morphology of BMSCs changed to neuron-like cells with a comprehensive axon-like connection with surrounding cells in (a) BDNF+ group (b) BDNF+, group and (c) BDNF- and group. BDNF+ group presented an increased number of neuron-like cells than the other groups. (d) Mock group. Scale bar, $100 \mu \mathrm{m}$. BMSCs, bone marrow-derived mesenchymal stem cells; BDNF, brain-derived neurotrophic factor.
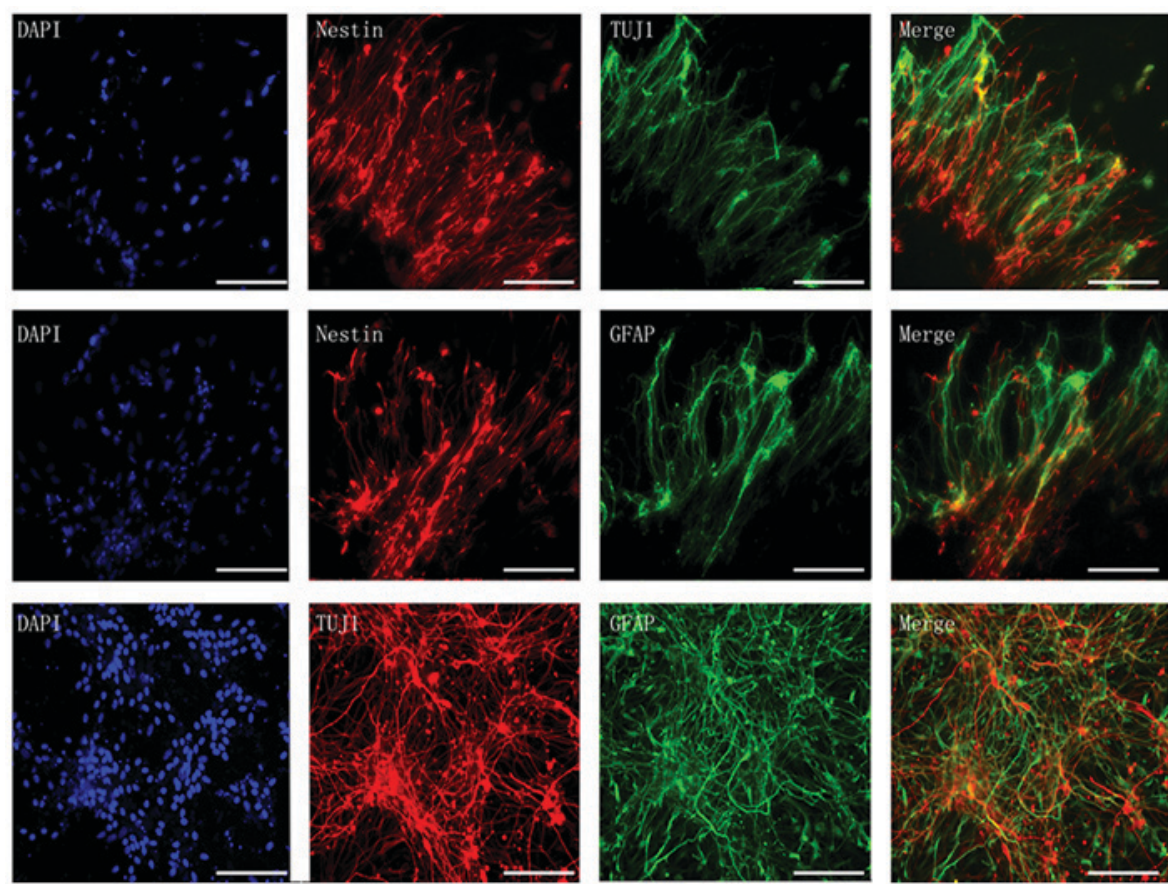
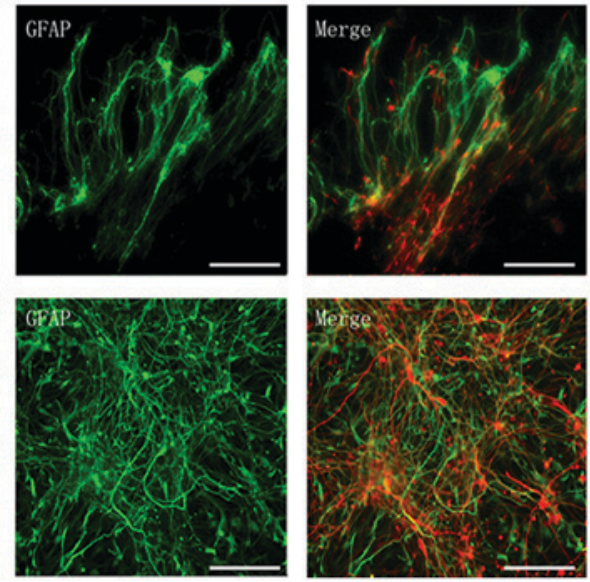

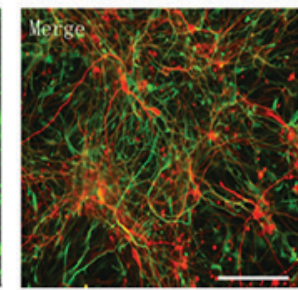

Figure 8. Immunofluorescence of BMSCs following neural differentiation in BDNF+ group. Immunofluorescence demonstrated positive counterstaining of nestin + TUJ1, nestin + GFAP and TUJ1 + GFAP in the BDNF+ group. Scale bar=50 $\mu \mathrm{m}$. BMSCs, bone marrow-derived mesenchymal stem cells; BDNF, brain-derived neurotrophic factor; TUJ1, class $3 \beta$ tubulin; GFAP, glial fibrillary acidic protein.

the foundation for cell regenerative therapy and cell paracrine effect-based therapy.

Previous studies have utilized genetically engineered MSCs modified using various gene therapy methods, including delivery using adenoviral transfer $(18,19)$, Lipofectamine (20), physical cell puncture (21), electroporation (22), soundwaves (23) and lentiviruses $(4,24)$. The primary advantage of lentivirus transfection is the capacity of gene integration with high efficiency, which establishes a new cell line that expresses the target gene (25). Initial vector transfection efficiency and optimization studies may provide the foundation of future in vivo experiments, and therefore the efficiency of vector transfection of MSCs may provide a pre- in vivo evaluation $(4,24,25)$. In canine MSCs, lentivirus mediated gene overexpression has been rarely reported (24). Previously, Ahn et al (26) used a lentivirus to overexpress interferon- $\beta$ in MSCs to treat canine melanoma, however the process of optimizing the MOI was not reported. Lentiviruses can infect proliferating and non-proliferating cells, which means that BMSCs can be eGFP positive but not viable. Therefore, cellular viability analysis is required to assess viral cytotoxicity and to optimize the MOI. In this study, time points for transfection were set at day 3,7 and 14, which is the duration usually required for expression of eGFP (12). The results of the present study demonstrated that, at day 7 , lentivirus transfection at MOI 10 resulted in a marked increase in eGFP expression and proliferation capacity, and this was maintained at day 14 .

BDNF is an essential molecule for cerebral nerve regeneration $(27,28)$. However, the underlying mechanism is not fully understood. A previous in vitro study demonstrated that a BDNF overexpression plasmid promoted neural growth through crosstalk with the Wnt/ $\beta$-catenin signaling pathway via glycogen synthase kinase-3 $\beta$ (29). Oh et al (30) reported that, following co-culture with MSCs, neuronal progenitor cells expressed significantly higher levels of neural markers, including GFAP and nestin, via activation of the Wnt signaling pathway. In this study, the expression of neural markers GFAP 
and nestin were also associated with neural differentiation, and BDNF-transfected MSCs exhibited higher expression of these markers. Additionally, the results also demonstrated increased expression of TUJ1 in cells overexpressing BDNF, which may be associated phosphorylation of protein kinase B (31). However, further studies focusing on the quantification of specific proteins that affect the signaling pathways are required.

Although not significant, the expression level of VEGF, which is an essential molecule involved in angiogenesis, was also increased by overexpression of BDNF. In addition, except for the increased expression of neurotropic genes (Nestin, TUJ1, GFAP and BDNF), the results demonstrated an increased gene expression of CXCR-4. CXCR-4 and its ligand, chemokine stromal cell-derived factor-1, have a critical role in MSC homing and recruitment at injury sites (32). This upregulation of CXCR-4 suggested that MSCs may actively migrate following neural differentiation in vivo.

The main limitation of this study is that only in vitro experiments were performed, and the neural differentiation efficiency and therapeutic effects of BDNF-modified BMSCs in vivo may be different due to the altered microenvironment. However, the present study demonstrated that lentivirus transfection is feasible for SPIO-labeled canine BMSCs, and overexpression of BDNF may contribute to the neural differentiation after successful transfection. This preliminary data may be important for further in vivo studies in large animals.

\section{Acknowledgements}

Not applicable.

\section{Funding}

The present study was supported by the National Natural Science Foundation of China (grant nos. 81571777, 81471764 and 81501565) and the Foundation of Research and Innovation Program for Postgraduates in Jiangsu Province (grant no. KYLX15_0957).

\section{Availability of data and materials}

The datasets used and/or analyzed during the current study are available from the corresponding author on reasonable request.

\section{Authors' contributions}

SL and HBS conceived and designed the experiments. XLL, QQZ and BW performed the experiments. BW, SSL and XQX analyzed the data. XLL wrote the manuscript which was revised and reviewed by QQZ, SSL and XQX, and the final version was approved by SL and HBS.

\section{Ethics approval and consent to participate}

All the animal experimental procedures were approved by the local Animal Experiment Ethical Committee (Nanjing Medical University, Nanjing, China).

\section{Consent for publication}

Not applicable.

\section{Competing interests}

The authors declare that they have no competing interests.

\section{References}

1. Volk SW, Wang Y and Hankenson KD: Effects of donor characteristics and ex vivo expansion on canine mesenchymal stem cell properties: Implications for MSC-based therapies. Cell Transplant 21: 2189-2200, 2012.

2. Golpanian S, Wolf A, Hatzistergos KE and Hare JM: Rebuilding the damaged heart: Mesenchymal stem cells, cell-based therapy, and engineered heart tissue. Physiol Rev 96: 1127-1168, 2016.

3. Baligar P, Mukherjee S, Kochat V, Rastogi A and Mukhopadhyay A: Molecular and cellular functions distinguish superior therapeutic efficiency of bone marrow CD45 cells over mesenchymal stem cells in liver cirrhosis. Stem Cells 34: 135-147, 2016.

4. Tseng TC and Hsu SH: Substrate-mediated nanoparticle/gene delivery to MSC spheroids and their applications in peripheral nerve regeneration. Biomaterials 35: 2630-2641, 2014.

5. Aggarwal S and Pittenger MF: Human mesenchymal stem cells modulate allogeneic immune cell responses. Blood 105: 1815-1822, 2005.

6. Kang SK, Shin IS, Ko MS, Jo JY and Ra JC: Journey of mesenchymal stem cells for homing: Strategies to enhance efficacy and safety of stem cell therapy. Stem Cells Int 2012: 342968, 2012.

7. Khubutiya MS, Vagabov AV, Temnov AA and Sklifas AN: Paracrine mechanisms of proliferative, anti-apoptotic and anti-inflammatory effects of mesenchymal stromal cells in models of acute organ injury. Cytotherapy 16: 579-585, 2014.

8. Burdon TJ, Paul A, Noiseux N, Prakash S and Shum-Tim D: Bone marrow stem cell derived paracrine factors for regenerative medicine: Current perspectives and therapeutic potential. Bone Marrow Res 2011: 207326, 2011.

9. Nagahara AH and Tuszynski MH: Potential therapeutic uses of BDNF in neurological and psychiatric disorders. Nat Rev Drug Discov 10: 209-219, 2011.

10. Kermani P and Hempstead B: Brain-derived neurotrophic factor: A newly described mediator of angiogenesis. Trends Cardiovasc Med 17: 140-143, 2007.

11. Rink C, Christoforidis G, Abduljalil A, Kontzialis M, Bergdall V, Roy S, Khanna S, Slivka A, Knopp M and Sen CK: Minimally invasive neuroradiologic model of preclinical transient middle cerebral artery occlusion in canines. Proc Natl Acad Sci USA 105: 14100-14105, 2008.

12. Lu SS, Liu S, Zu QQ, Xu XQ, Yu J, Wang JW, Zhang Y and Shi HB: In vivo MR imaging of intraarterially delivered magnetically labeled mesenchymal stem cells in a canine stroke model. PLoS One 8: e54963, 2013.

13. Xu W, Chen J, Liu X, Li H, Qi X and Guo X: Autologous bone marrow stromal cell transplantation as a treatment for acute radiation enteritis induced by a moderate dose of radiation in dogs. Transl Res 171: 38-51, 2016.

14. Penha EM, Meira CS, Guimarães ET, Mendonça MV, Gravely FA, Pinheiro CM, Pinheiro TM, Barrouin-Melo SM, Ribeiro-Dos-Santos R and Soares MB: Use of autologous mesenchymal stem cells derived from bone marrow for the treatment of naturally injured spinal cord in dogs. Stem Cells Int 2014: 437521, 2014.

15. Woodbury D, Schwarz EJ, Prockop DJ and Black IB: Adult rat and human bone marrow stromal cells differentiate into neurons. J Neurosci Res 61: 364-370, 2000.

16. Wei L, Fraser JL, Lu ZY, Hu X and Yu SP: Transplantation of hypoxia preconditioned bone marrow mesenchymal stem cells enhances angiogenesis and neurogenesis after cerebral ischemia in rats. Neurobiol Dis 46: 635-645, 2012.

17. Livak KJ and Schmittgen TD: Analysis of relative gene expression data using real-time quantitative PCR and the 2(-Delta Delta C(T)) method. Methods 25: 402-408, 2001. 
18. Hammer K, Kazcorowski A, Liu L, Behr M, Schemmer P, Herr I and Nettelbeck DM: Engineered adenoviruses combine enhanced oncolysis with improved virus production by mesenchymal stromal carrier cells. Int J Cancer 137: 978-990, 2015.

19. Kim MD, Kim SS, Cha HY, Jang SH, Chang DY, Kim W, Suh-Kim H and Lee JH: Therapeutic effect of hepatocyte growth factor-secreting mesenchymal stem cells in a rat model of liver fibrosis. Exp Mol Med 46: e110, 2014.

20. Ribeiro SC, Mendes R, Madeira C, Monteiro GA, da Silva CL and Cabral JM: A quantitative method to evaluate mesenchymal stem cell lipofection using real-time PCR. Biotechnol Prog 26: 1501-1504, 2010.

21. Han SW, Nakamura C, Kotobuki N, Obataya I, Ohgushi H, Nagamune T and Miyake J: High-efficiency DNA injection into a single human mesenchymal stem cell using a nanoneedle and atomic force microscopy. Nanomedicine 4: 215-225, 2008

22. Liew A, André FM, Lesueur LL, De Ménorval MA, O'Brien T and Mir LM: Robust, efficient, and practical electrogene transfer method for human mesenchymal stem cells using square electric pulses. Hum Gene Ther Methods 24: 289-297, 2013.

23. Choi Y,Park JE, Jeong JS, Park JK, Kim J and Jeon S: Sound waves induce neural differentiation of human bone marrow-derived mesenchymal stem cells via ryanodine receptor-induced calcium release and Pyk2 activation. Appl Biochem Biotechnol 180: 682-694, 2016

24. McGinley L, McMahon J, Strappe P, Barry F, Murphy M, O'Toole D and O'Brien T: Lentiviral vector mediated modification of mesenchymal stem cells \& enhanced survival in an in vitro model of ischaemia. Stem Cell Res Ther 2: 12, 2011.

25. Han SH, Jang G, Bae BK, Han SM, Koh YR, Ahn JO, Jung WS, Kang SK, Ra JC, Lee HW and Youn HY: Effect of ectopic OCT4 expression on canine adipose tissue-derived mesenchymal stem cell proliferation. Cell Biol Int 38: 1163-1173, 2014.
26. Ahn Jo, Lee Hw, Seo Kw, Kang Sk, Ra Jc and Youn Hy: Anti-tumor effect of adipose tissue derived-mesenchymal stem cells expressing interferon- $\beta$ and treatment with cisplatin in a xenograft mouse model for canine melanoma. PLoS One 8: e74897, 2013.

27. Fouda AY, Alhusban A, Ishrat T, Pillai B, Eldahshan W, Waller JL, Ergul A and Fagan SC: Brain-derived neurotrophic factor knockdown blocks the angiogenic and protective effects of angiotensin modulation after experimental stroke. Mol Neurobiol 54: 661-670, 2017.

28. Helm EE, Tyrell CM, Pohlig RT, Brady LD and Reisman DS: The presence of a single-nucleotide polymorphism in the BDNF gene affects the rate of locomotor adaptation after stroke. Exp Brain Res 234: 341-351, 2016

29. Yang JW, Ru J, Ma W, Gao Y, Liang Z, Liu J, Guo JH and Li LY: BDNF promotes the growth of human neurons through crosstalk with the Wnt/ $\beta$-catenin signaling pathway via GSK-3 $\beta$. Neuropeptides 54: 35-46, 2015.

30. Oh SH, Kim HN, Park HJ, Shin JY and Lee PH: Mesenchymal stem cells increase hippocampal neurogenesis and neuronal differentiation by enhancing the wnt signaling pathway in an Alzheimer's disease model. Cell Transplant 24: 1097-1109, 2015.

31. Rahmani A, Kheradmand D, Keyhanvar P, Shoae-Hassani A and Darbandi-Azar A: Neurogenesis and increase in differentiated neural cell survival via phosphorylation of Akt1 after fluoxetine treatment of stem cells. Biomed Res Int 2013: 582526, 2013.

32. Li Y, Huang J, He X, Tang G, Tang YH, Liu Y, Lin X, Lu Y, Yang GY and Wang Y: Postacute stromal cell-derived factor-1a expression promotes neurovascular recovery in ischemic mice. Stroke 45: 1822-1829, 2014 